\title{
MANAGEMENT OF POSTOPERATIVE DISCITIS: A STUDY ON 20 CASES OF PROLAPSED LUMBER INTERVERTEBRAL DISC (PLID) OPERATION IN A TERTIARY LEVEL HOSPITAL IN BANGLADESH
}

\author{
DAS $\mathrm{S}^{1}$, MAHMOOD E ${ }^{2}$, ALAM MJ $^{3}$, RASHID MM ${ }^{4}$, MITRA PK
}

\begin{abstract}
:
Context: The incidence of postoperative discitis is relatively uncommon. The paucity of physical examination findings with a high index of suspicion in any patient presenting with increasing back pain after prolapsed lumber intervertebral disc (PLID) operation gives clue of diagnosis.

Purpose: To see the incidence, pathophysiology, laboratory markers and imaging finding of postoperative discitis, at the same time recommendation on treatment strategies with long term clinical outcome of discitis patient in Dhaka Medical College Hospital (DMCH), a tertiary level hospital in Dhaka, Bangladesh.

Methods: In this observational study, 20 patients were recruited who underwent PLID operation in the Department of Neurosurgery, DMCH, Dhaka, from January 2012 to June 2014. After preliminary selection of patients a specific protocol was followed, by taking history, clinical examination, biochemical parameters and neuroimaging. Result of treatment was observed on the basis of symptomatic improvement, with laboratory and imaging findings.

Results: The most common etiological agent is staphylococcus aureus. The C-reactive protein is the most sensitive clinical laboratory marker to assess the presence of infection and effectiveness of treatment response. MRI is the imaging modality of choice in the diagnosis of discitis (100\%). The majority of patients are managed adequately with culture specific antibiotics and immobilization with good long term outcomes (75\%). Operative intervention in those patients who fails to respond to medical treatment (25\%).

Conclusion: Postoperative discitis is a rare complication after PLID operation. Its diagnosis needs high index of suspicion, appropriate laboratory and imaging studies helps in diagnosis. In the majority of patients antibiotics and spinal immobilization has been shown to produce good long term outcomes. Operative intervention is rarely necessary in patients failing conservative treatment.
\end{abstract}

Keywords: Postoperative discitis; C-reactive protein; PLID (Prolapsed lumber intervertebral disc).

J Dhaka Med Coll. 2014; 23(2) : 186-190.

\section{Introduction}

Discitis is an infection of disc space following surgery. It is actually primary infection of the nucleus pulposus with secondary involvement cartilaginous end plate and vertebral body. The rare complication of post surgical discitis was first described as a clinical entity Ley Turnbull in $1953^{1}$. The incidence of post surgical discitis is approximately $0.2 \%$.

Pain is the common symptom of discitis, which is constant pain that becomes worse at night but up to $15 \%$ of patients may be pain free ${ }^{2}$. The patient has an initial post surgical relief of pain which is followed by a gradual increase of back pain ranging from mild to severe, return of their original pre-operative symptoms. The most common time frame for the development of increasing back pain is 1 to 4 weeks in the post operative period (ranging 2 days to 10 weeks $)^{3}$. The back pain is often out of proportion to any physical findings and is often accompanied with referred pain radiating into the buttocks, thighs, legs, groin, perineum or the abdomen.

Fever is less common, occurs in about $50 \%$ of patients ${ }^{4}$. Only in rare cases patient complaint of constitutional symptoms, such as fatigue or malaise and even rare is the presentation of

1. Dr. Sukriti Das, Assistant Professor, Department of Neurosurgery, Dhaka Medical College \& Hospital, Dhaka.

2. Prof. Ehsan Mahmood, Professor and Head, Department of Neurosurgery, Dhaka Medical College \& Hospital, Dhaka.

3. Dr. Md. Jahangir Alam, Neurosurgeon, Department of Neurosurgery, Dhaka Medical College Hospital, Dhaka.

4. Dr. Md. Mamunur Rashid, MS- resident, Department of Neurosurgery, Dhaka Medical College, Dhaka.

5. Dr. Pijush Kumar Mitra, Medical Officer, Department of Neurosurgery, Dhaka Medical College Hospital, Dhaka. Correspondence: Dr. Sukriti Das, Assistant Professor, Department of Neurosurgery, Dhaka Medical College \& Hospital, Dhaka. Cell Phone: +8801711676848, Email: sukriti66@yahoo.com 
sepsis with high fevers, chills and sweats probably due to aggressive organism or a florid infections process that frequently warrants an aggressive workup. Neurological deficit like lower limbs paresis / paralysis, sensory deficit, rediculopathy and sphincter dysfunction are uncommon. The surgical skin incision is normal in the vast majority of cases because fewer than $10 \%$ will show signs of incisional infection, including erythema, dehiscense and/ or purulence ${ }^{3}$. Discitis is a serious complication which can cause morbidity and mortality $(0,06 \%)$ more often due to complications like septicaemia, MI, pneumonia and embolism ${ }^{5}$.

Several factors have been claimed to play role in the pathogenesis of discitis. The patient related factors are: age, trauma, diabetes, malnutrition, immunosuppression, malignancy, obesity, smoking and extended hospital stay etc. ${ }^{6}$. The operation related factors are: OT environment, OT asepsis, instrument and linen autoclave system, number of person in OT, OT stuff cleanliness, use of C-arm and microscope, use of prophylactic antibiotics, over use of monopolar diathermy, haemostasis, muscles laceration and bleeding, use of cottonoid, local steroid, antibiotics and anesthetic agents, blood transfusion, serial OT list. Wide ranges of organism have been associated with postsurgical discitis, among them most common infection etiologic agent is Staphylococcus aureus followed by Staphylococcus species and anaerobic organisms. Other less common organisms include Streptococcus viridians, E. coli, Pseudomonas aeruginosa, Mycobacterium tuberculosis, fungus and others. Possible source of microbial infection which direct inoculate bacteria at the time of surgery or soiling of the incision in the fresh post-operative phase of through haematogenous spread ${ }^{7}$. Prophylactic antibiotics have clearly established as an effective adjunct to decrease the chance of a surgical site infection ${ }^{7}$.

Infection rate varies with the type of surgery and site of surgery. In case of PLID discectomy operation in lumbo-sacral region, the incidence of post surgical discitis is $0.5 \%$ to $5 \%{ }^{9}$. The incidence of surgical site infection after decompression laminectomy, discectomy and fusion is quoted to be $3 \%$ or even lower but the incidence increases to as high as $12 \%$ with addition of instrumentation.
In another study discectomy has infection rates ranging from $0.5 \%$ to $5 \%{ }^{10}$. Fusion without instrumentation associated with infection rates from $0.4 \%$ to $4.3 \%$ and the use of internal fixator raises the risk of discitis significantly, $6.6 \%$ to $8.7 \%{ }^{11}$.

The diagnosis cannot often be established in a timely fashion based on the history, physical examination, laboratory study like- ESR, Creactive protein and blood culture and imaging studies like plain radiographs, magnetic resonance imaging, computed tomography and radionuclide scanning. C-reactive protein \& ESR are the most sensitive clinical laboratory maker to assess the presence of infection and effectiveness of treatment response ${ }^{12}$. MRI is the imaging modality of choice in the diagnosis of spinal infection/discitis.

The majority of patients are managed adequately with culture specific antibiotics and spinal immobilization with good long term outcomes. Operative intervention debridement followed by antibiotics treatment in patient who fail to respond to non-operative treatment. Widespread use of pre-operative prophylactic antibiotics substantially reduced the incidence of post-operative infections complications after spinal surgery ${ }^{13}$. Post-operative incidence of discitis is lower in those patients whom prophylactic antibiotics were used than those who received no antibiotics at all. Postoperative established discitis has no universally accepted treatment protocol, management decision vary among surgeons and regions of the country. Majority author believe that immobilization with orthosis with intravenous administration of specific antibiotics for six weeks followed by additional 6 weeks of oral antibiotics ${ }^{14}$. If organism is not determined, broad-spectrum antibiotics with anti-staphylococcal coverage are recommended. If constitutional symptoms progress, back pain worsens or the laboratory indexes continue to worsen, an open procedure may be considered for debridement and/ or fusion and identification of organisms. In the adult patient, surgical intervention is often reserved in cases with large paraspinal abscesses or lack of improvement with immobilization and antibiotic treatment. Open surgical biopsies have a much higher positive culture rate (up to $100 \%$ ) and autograft fusion often heals predictably with adequate immobilization. 


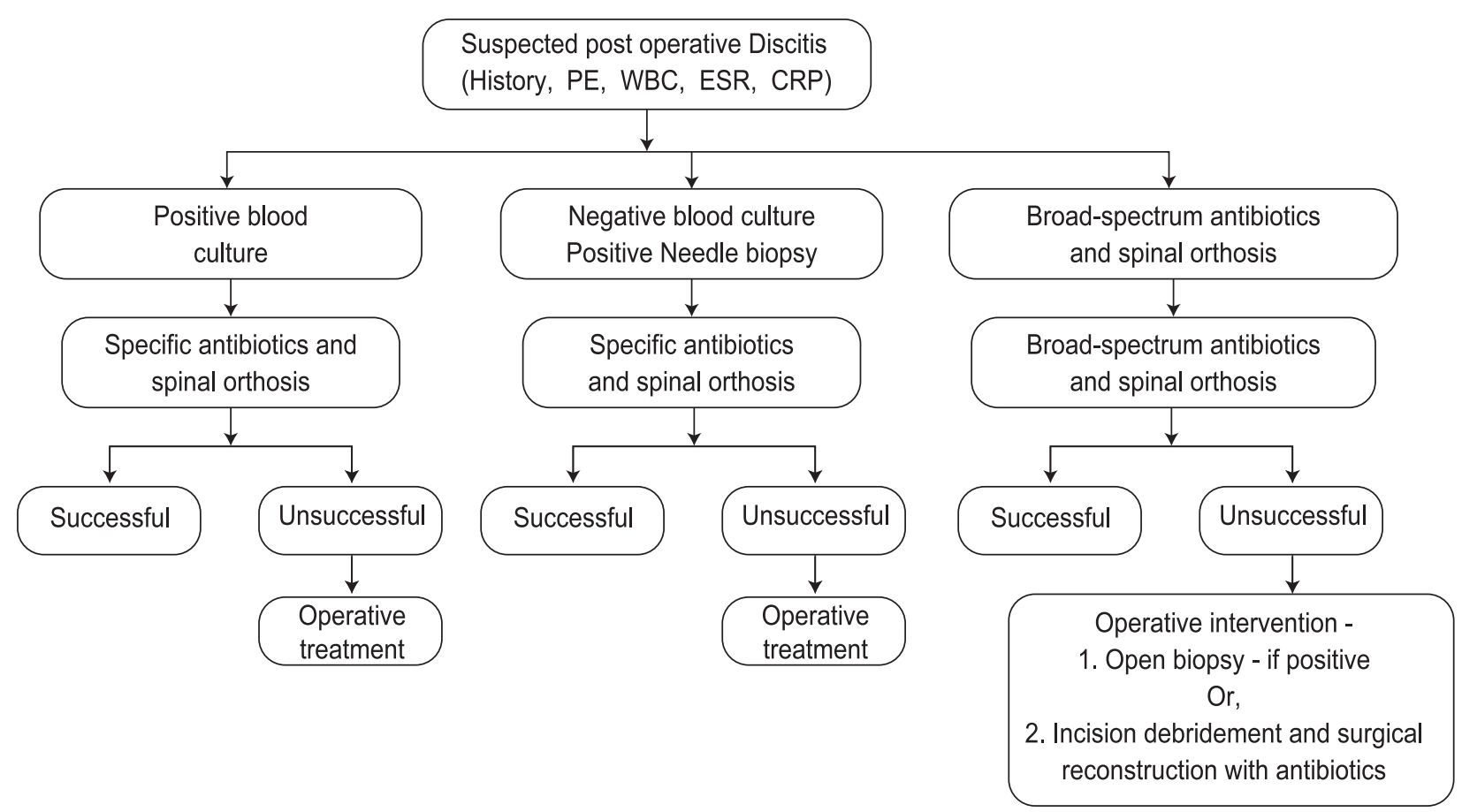

Fig.-1: Post-operative discitis management algorithm.

$(\mathrm{PE}=$ Physical examination, $\mathrm{WBC}=$ White blood count, $\mathrm{ESR}=$ Erythrocyte sedimentation rate, $\mathrm{CRP}=$ C-reactive protein) ${ }^{15}$

\section{Methods}

It is prospective observational study, conducted in the department of Neurosurgery, Dhaka Medical College Hospital during the period of January 2012 to June 2014, and 20 of patients who developed discitis after PLID operation discectomy among 180 were taken for the study.

\section{Results:}

The results are stated below:

Age distribution:

\begin{tabular}{lc}
\hline Years & No. of Patients \\
\hline $20-30$ years & 6 \\
$31-40$ years & 8 \\
$41-50$ years & 4 \\
$>50$ years & 2 \\
\hline
\end{tabular}

Sex distribution:

Male: Female $=14: 6$

Patient related risk factors:

\begin{tabular}{lc}
\hline Diabetes mellitus & 6 \\
Obesity & 5 \\
Immunosuppressive drugs & 1 \\
Smoking & 10 \\
Malnutrition & 2 \\
\hline
\end{tabular}

Per-operative procedural complications:

\begin{tabular}{lcc}
\hline Unipolar diathermy burn & 12 & $60 \%$ \\
\hline Curettage of end plate & 6 & $30 \%$ \\
Use of local antibiotics & 10 & $50 \%$ \\
Use of local steroid & 6 & $30 \%$ \\
Use of cottonoid & 8 & $40 \%$ \\
\hline
\end{tabular}

Clinical presentation of patients who developed discitis:

Persistent pain with/without $\quad 100 \%$

neurological deficit

Relation of pain with movement $\quad 90 \%$

Fever with chills $\quad 90 \%$

Tenderness over spine $\quad 90 \%$

Paravertebral muscle spasm $\quad 90 \%$

Difficulty in walking $\quad 80 \%$

Superficial surgical site infection $\quad 60 \%$

(SSSI)

Impairment of sensation of leg $\quad 10 \%$

Impairment of bladder and bowel $\quad 10 \%$ 


\section{Biochemical parameters:}

Comparison of biochemical parameters between patients with and without discitis shows that ESR and serum level of C-reactive protein were significantly higher in patients with discitis $(100 \%)$

- $\quad X$-ray findings shows- Narrowing of disc space with end plate irregularities in 100\% and $95 \%$ cases respectively.

- $\quad$ MRI findings- MRI findings showed signs of florid inflammation in all cases, Spondylodiscitis in 95\% and spinal epidural abscess in $5 \%$. In post contrast MRI showed homogenous enhancement of lesion in $95 \%$.

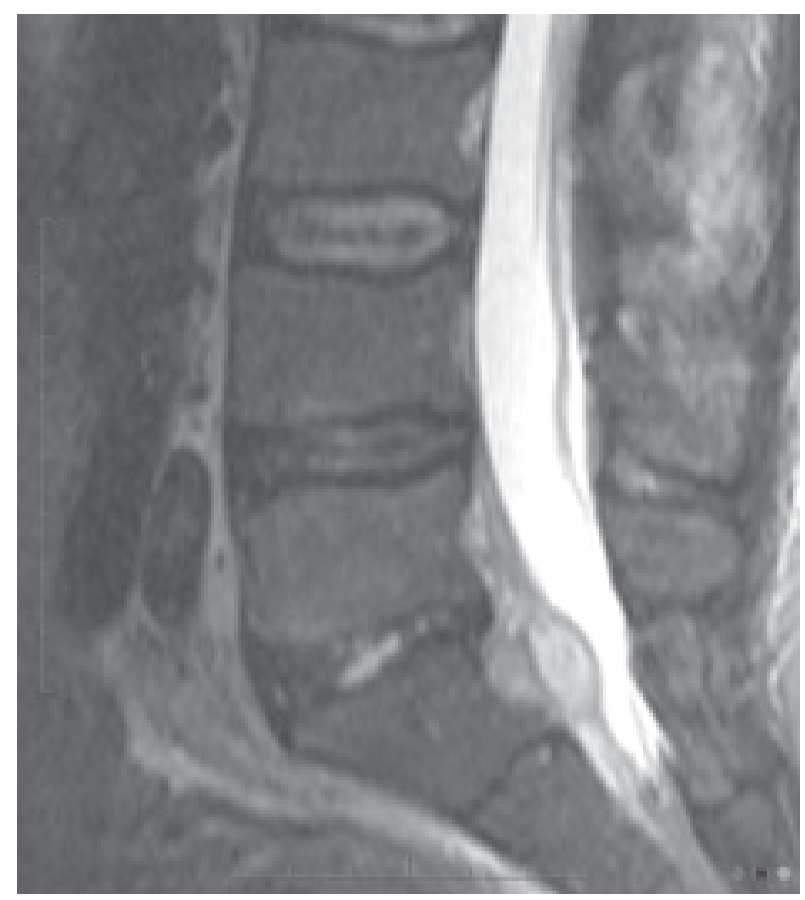

Fig. 2: MRI showing postoperative discitis with retrodiscal abscess.

\section{Post-operative discitis: treatment options:}

1. Antibiotics + Immobilization in 15 patients (75\%).

2. Re-exploration/ Surgical intervention in case of large paraspinal \& intervertebral abscess in 4 patients $(20 \%)$.

3. In postoperative discitis \&spinal instability: Trans pedicular screw fixation and bony fusion in 1 patient $(5 \%)$.

\section{Prognosis:}

Fortunately, the overall long term prognosis after non operative treatment for post operative discitis is quiet good.Our studytshown that $90 \%$ of patients are pain free after resolution of the infection, with $75 \%$ going on to either a bony ankylosis or stable fibrous union. However, $100 \%$ patient relief symptoms after surgical intervention. Surgical intervention and specific antibiotics therapy gives better outcome than only antibiotics.

\section{Conclusion:}

In the majority of the patients (75\%), a nonsurgical approach including absolute bed rest, spinal bracing and culture, specific antibiotic is needed for eradication of the disc space infection with good long term clinical outcome. Those patients fail to respond conservatively, leading to continued infection, pain or deformity, they underwent operative intervention (25\%) and had symptomatic relief.

\section{References:}

1. Turnbull F. Postoperative inflammatory disease of lumbar discs. J Neurosurg 1953; 10: 469-73.

2. Torda AJ, Gottlieb T, Bradbury R. Pyogenic vertebral osteomyelitis: analysis of 20 cases and review. Clin Infect Dis 1995; 20: 320-8.

3. Rawlings CE, Wilkins RH, Gallis HA, Goldner $\mathrm{JL}$, Francis R. Post-operative intervertebral disc space infection. Neurosurgery 1983; 13: 371-5.

4. Mylona E, Samarkos M, Kakalou E, Fanourgiakis $\mathrm{P}$, Skoutelis A. Pyogenic vertebral osteomyelitis : a systemic review of clinical characteristics, Semim Arthritis Rheum 2009; 39: 10-7.

5. Samandouras G Apuzzo MLJ. The Neurosurgeons Handbook. Oxford: Oxford University Press; 2010 .

6. Rechtine GR, Bono PL, Cahill D, Bolesta MJ, Chrin AM. Postoperative wound infection after instrumentation of thoracic and lumbar fractures. J Orthop Trauma 2001; 15: pp. 566-9.

7. Dubousset J, Shufflebarger H, Wenger D. Late infection with CD instrumentation. Orthop Trans 1994; 18: 121.

8. Keller RB, Pappas AM. Infection after spinal fusion using internal fixation instrumentation. Orthop Clin North Am 1972; 3: 99-111.

9. Bassewitz\& HL, Fishgrund JS, Herkowitz HN. Postoperative spine infections. Semin Spine Surg 2000; 12: 203-11. 
10. Volmer DG, Tandon N. Infection of the spine. In: Winn HR. Youman's Neurological Surgery. $5^{\text {th }}$ edn. Philadelphia: Elsevere Saunders; 2011.

11. Massie JB, Heller JG, Abitbol JJ, McPherson D, Garfin SR. Postoperative posterior spinal wound infections. Clin Orthop 1992; 284: 99-108.

12. Roberts FJ, Walsh A, Wing P. The influence of surveillance methods on surgical wound infection rates in a tertiary care spinal surgery service. Spine 1998; 23: 366-70.
13. El-Gindi S, Aref S, Salama M, Andrew J, Infection of intervertebral discs after operation. J Bone Joint Surg 1976; 58B: 114-6.

14. Fouquet B, Goupille P, Jattiot F, et al. Discitis after lumbar disc surgery, features of "aseptic" and "septic" forms. Spine 1992; 17: 356-8.

15. Silber JS, Anderson G, Vaccaro AR, Anderson PA, McCormick P. Management of postprocedural discitis. Spine 2002; 279-87. 